Revue des patrimoines

$36 \mid 2018$

Les archives photographiques de presse, pratiques comparées et enjeux méthodologiques

\title{
Le fonds Jean Lattes : une archive photographique en construction
}

The Jean Lattes collection, a photographic archive in the making

Angelina Meslem, Wilfrid Eon et Clémentine Vialar

\section{(2) OpenEdition}

Journals

Édition électronique

URL : http://journals.openedition.org/insitu/17738

DOI : $10.4000 /$ insitu. 17738

ISSN : 1630-7305

Éditeur

Ministère de la culture

Référence électronique

Angelina Meslem, Wilfrid Eon et Clémentine Vialar, « Le fonds Jean Lattes : une archive

photographique en construction », In Situ [En ligne], 36 | 2018, mis en ligne le 15 octobre 2018,

consulté le 01 mai 2019. URL : http://journals.openedition.org/insitu/17738 ; DOI : 10.4000/

insitu. 17738

Ce document a été généré automatiquement le 1 mai 2019.

\section{(†) $\odot$

In Situ Revues des patrimoines est mis à disposition selon les termes de la licence Creative Commons Attribution - Pas d'Utilisation Commerciale - Pas de Modification 4.0 International. 


\section{Le fonds Jean Lattes : une archive photographique en construction}

The Jean Lattes collection, a photographic archive in the making

Angelina Meslem, Wilfrid Eon et Clémentine Vialar

Les auteurs remercient chaleureusement Gilles Walusinski pour ses précieuses explications et sa disponibilité.

1 Cet article se propose d'aborder l'étude d'un fonds du photographe de presse Jean Lattes (1917-1996), constitué de près de 200000 clichés $^{1}$, auxquels s'ajoutent des planchescontact, quelques tirages, des inventaires et des archives personnelles. Don de Janine Lattes, son épouse, en 2011, aux Archives départementales des Yvelines (Saint-Quentinen-Yvelines), ce fonds constitue un témoignage remarquable sur la pratique d'un photographe qui a mené une carrière sous un double statut, à la fois pigiste pour plusieurs journaux et participant du projet collectif de deux agences photographiques françaises notables, Gamma et Viva (fig. 1). 
Figure 1

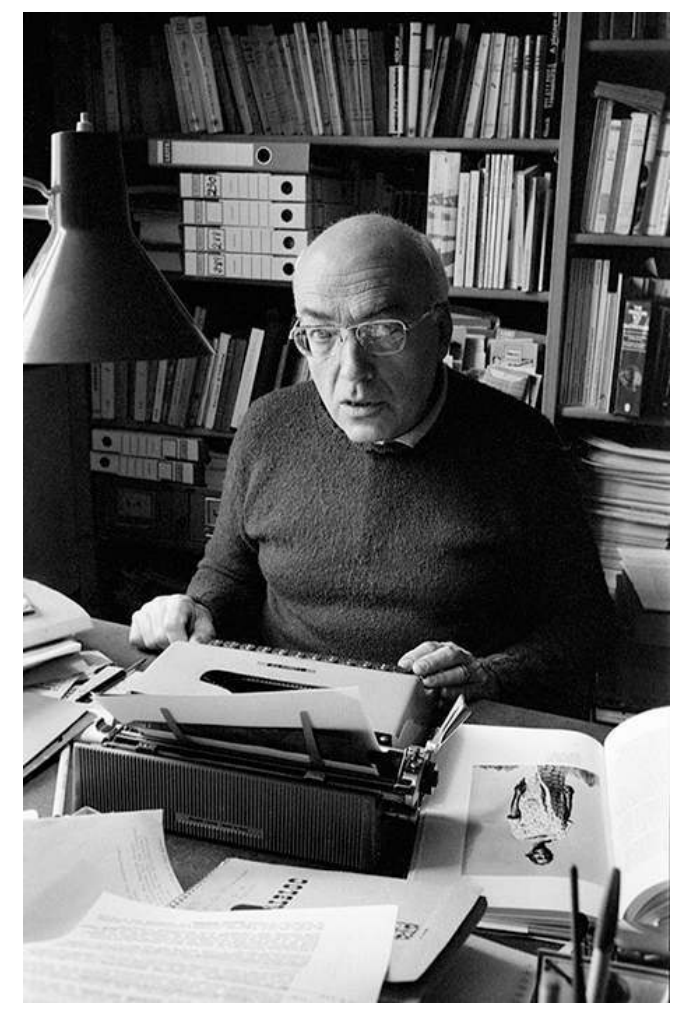

Portrait de Jean Lattes, Le Vésinet, 1976.

PHOT. GILLES WALUSINSKI. @ GILLES WALUSINSKI, 1976.

Le traitement intellectuel de ce type de donation est une première pour les archivistes des Yvelines, de sorte qu'elle a suscité plusieurs interrogations. Quelles orientations prendre en termes d'inventaire, de signalement, de restauration ou de conditionnement sur des objets patrimoniaux multiformes? Comment enrichir la réflexion par la compréhension fine du contexte de production quand les éléments biographiques sont transmis de façon fragmentaire au moment du don? Si des fonds photographiques y sont déjà présents, aucun d'eux n'a jusqu'ici bénéficié d'un traitement spécifique ${ }^{2}$. Comme le souligne Patrice Marcilloux ${ }^{3}$, l'absence d'une terminologie commune pour désigner les fonds d'images fixes et d'images animées dans les archives n'est certainement pas étrangère à la difficulté de repérer les fonds. Un obstacle supplémentaire est à signaler : la variété des procédés photographiques et les difficultés d'identification pour un nonspécialiste du domaine $e^{4}$. De même, alors que les bibliothèques sont dotées d'un manuel pour le traitement des documents iconographiques, Images et bibliothèque ${ }^{5}$, les archivistes en sont dépourvus. Cette approche réflexive sur le fonds Lattes sera sans aucun doute bénéfique pour le traitement des autres fonds photographiques et dans la perspective de versements futurs.

Alors que des tirages de presse circulent sur le marché de l'art, s'exposent et intègrent les collections patrimoniales des musées ${ }^{6}$, ce qui renouvelle leur statut, l'intégration d'un riche fonds de négatifs en archives n'est pas sans poser des questions différentes, car si les photographies partagent le problème de la conservation et du conditionnement, la numérisation et l'indexation des clichés soulèvent des problématiques spécifiques. 


\section{Des archives orphelines en péril}

4 Un fonds photographique de presse entre dans la catégorie des archives «orphelines ", c'est-à-dire celles issues de la sphère privée qui n'ont pas d'entrée institutionnelle automatique au sein d'un service public d'archives ${ }^{7}$. Soucieuse de sauver l'œuvre de Jean Lattes, son défunt mari, Janine Lattes avait pris, à la fin des années 2000, plusieurs attaches avant de trouver auprès de la Mission de la photographie du ministère de la Culture un soutien pour l'accompagner dans ses démarches et trouver un lieu d'accueil. Le conseil départemental (ancien conseil général) des Yvelines, département où résidaient les Lattes depuis quarante ans, disposait d'un bâtiment d'archives pas encore saturé. Lorsque sa directrice, Élisabeth Gautier-Desvaux, avec le soutien du président du conseil général Alain Schmitz, s'engage à recevoir ce fonds volumineux, deux courses contre la montre débutent : la première concerne la ratification d'une convention écrite portant sur les modalités d'entrée et surtout les droits d'utilisation entre le futur détenteur et les ayants droit dont l'âge avancé pouvait à tout moment remettre en cause les premiers accords verbaux ; la seconde, le « sauvetage » de l'ensemble photographique, qui commençait à se détériorer, et sa documentation.

5 Janine Lattes, dernière femme du photographe, avait affiché dès l'origine son intention de céder l'ensemble de ses droits. Sans enfant, elle n'en détenait cependant que l'usufruit ; la nue-propriété revenait à Pierre Lattes, fils unique de Jean et de sa première femme, Aline. La brouille entre le fils et le père à la fin de sa vie aurait pu être un obstacle à cet accord, mais la détermination de Janine Lattes, aidée dans cette entreprise par la Mission de la photographie ${ }^{8}$, permit d'aboutir en moins d'un an à un accord. Le 18 juin 2012, le conseil départemental des Yvelines obtint officiellement la propriété pleine et entière du fonds ainsi que les droits d'exploitation.

6 Les photographies entreposées dans le bureau de Jean Lattes, dans sa maison du Vésinet, furent transférées aux archives départementales des Yvelines dès 2011. La priorité consista alors à évaluer le fonds, à établir un état sanitaire et à le comprendre avec l'aide des proches du photographe. Rien ne se passa malheureusement comme prévu. Le départ à la retraite des principaux acteurs du projet, côté archives, et le décès de Pierre Lattes, en 2013, suivi de celui de Janine Lattes un an après privèrent la génération suivante de témoignages pouvant aider à comprendre, documenter et traiter cet ensemble volumineux ${ }^{9}$ (fig. 2). 
Figure 2

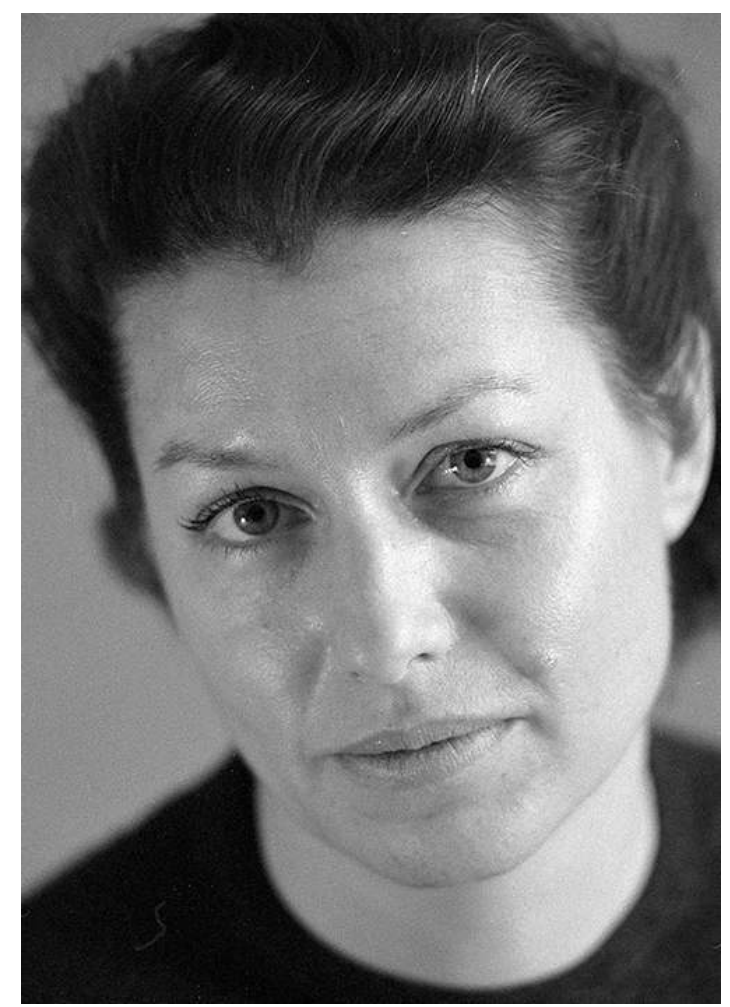

Portrait de Janine Lattes, Le Vésinet, 1976.

Phot. Gilles Lattes. (c) Archives départementales des Yvelines, 2016.

7 Par ailleurs, l'état physique des négatifs suscitait des inquiétudes. Impression de gras au toucher, odeur âcre et piquante et décollement de couches pour une centaine de clichés : autant d'indices de cette détérioration presque inéluctable à laquelle sont voués les documents argentiques ${ }^{10}$. En 2015, une étude sanitaire des films négatifs (acétate) commandée à des restaurateurs de photographies révèle une dégradation de 15 à $20 \%$ des supports selon les ensembles. Conserver les films dans une chambre froide stopperait certes ce phénomène mais dès lors, les supports plus sensibles aux chocs thermiques ne pourraient plus être sortis pour être communiqués sans risquer d'accélérer leur dégradation. De telles mesures de conservation s'imposaient comme l'étape finale dans la chaîne de traitement qui se dessinait.

\section{Le classement méthodique des Lattes}

8 Malgré une collecte lacunaire d'informations au moment de la donation, le cadre performant du classement est assez aisément compréhensible. Aidé de sa femme, Jean Lattes a soigneusement classé, numéroté, rangé les photographies en élaborant des outils d'accès plus ou moins développés et adaptés à ses besoins de photographe. Même si ceuxci semblent parfois trop peu détaillés ou exhaustifs pour une personne extérieure, ils permettent de saisir l'organisation de la production photographique. Ainsi, trois ensembles se distinguent, correspondant aux trois périodes de la vie du photographe telles qu'il les décrit dans son autobiographie ${ }^{11}$. 
Le premier ensemble est constitué de 6193 négatifs noir et blanc $(6$ x 6) réalisés de 1949 à 1954 avec un "Rolleiflex acheté d'occasion ", pour le journal France Dimanche auquel il collabore au début de sa carrière de photojournaliste. Conservés dans des enveloppes par numéro de publication, ces reportages sont répertoriés dans un inventaire thématique (fig. 3). Cette production est complétée par environ 600 négatifs noir et blanc que le photographe a rangés et inventoriés séparément. Classés par thème, les clichés sont de même type et datent de la même époque. Ces éléments laissent supposer qu'il s'agit de reportages réalisés pour France Dimanche mais non publiés, hypothèse qui devra être confortée par une étude approfondie de ces photographies.

Figure 3

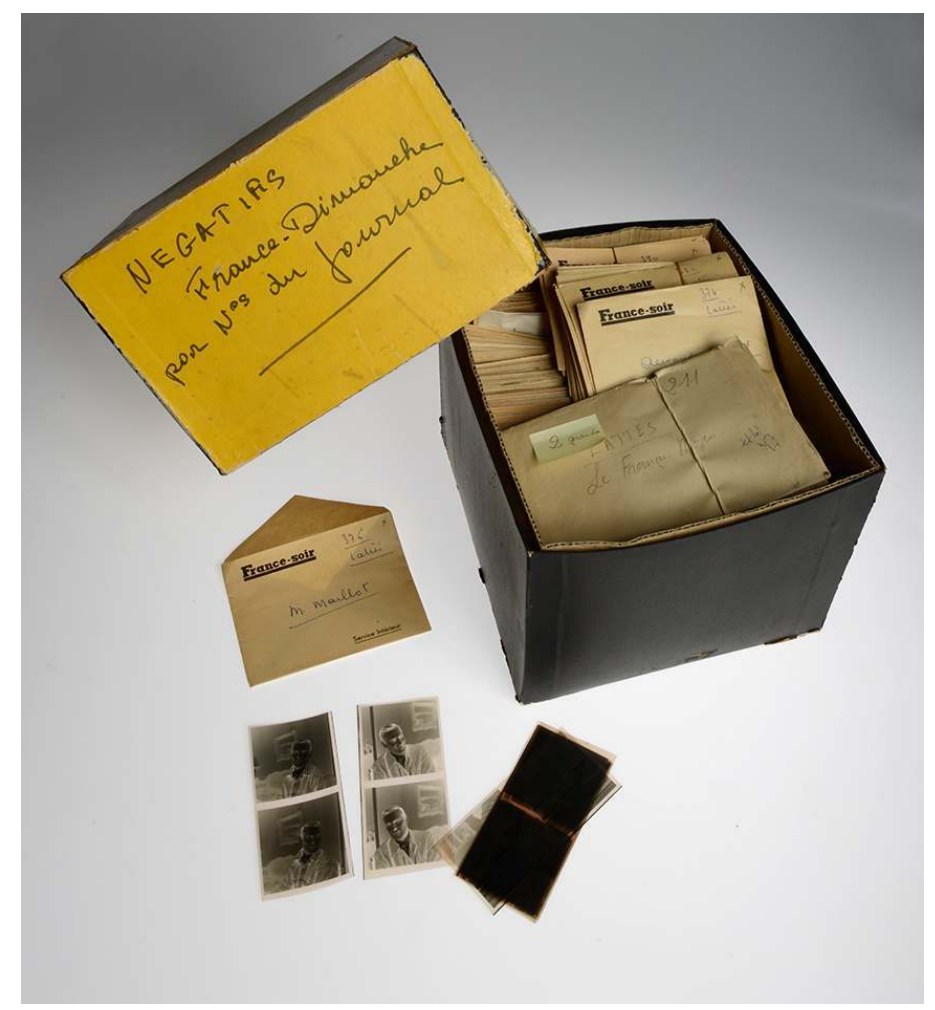

Négatifs publiés dans France Dimanche, fonds Jean Lattes (40 Fl).

Phot. Jean-Bernard Barsamian. (C) Archives départementales des Yvelines, 2015.

10 Le deuxième, de loin le plus volumineux, correspond à son travail en tant que photographe indépendant, à partir de 1954 et jusqu'à sa mort ${ }^{12}$. Durant cette période, Jean Lattes collabore régulièrement à des journaux comme Elle, Science et Vie, L'Express, Francesoir et à des publications étrangères telles que Time, Life et Look comme en attestent ses carnets de commande.

11 Cette collection comporte environ 130000 négatifs noirs et blancs ( $24 \times 36)^{13}$ auxquels sont associées 5181 planches-contact ainsi que 32000 diapositives couleurs, 68 bandes de négatifs couleur et une centaine de phototypes divers: tirages positifs $9 \times 12$, Ektachromes couleur 9 x 12 à diapositives noir et blanc (fig. 4). 
Figure 4

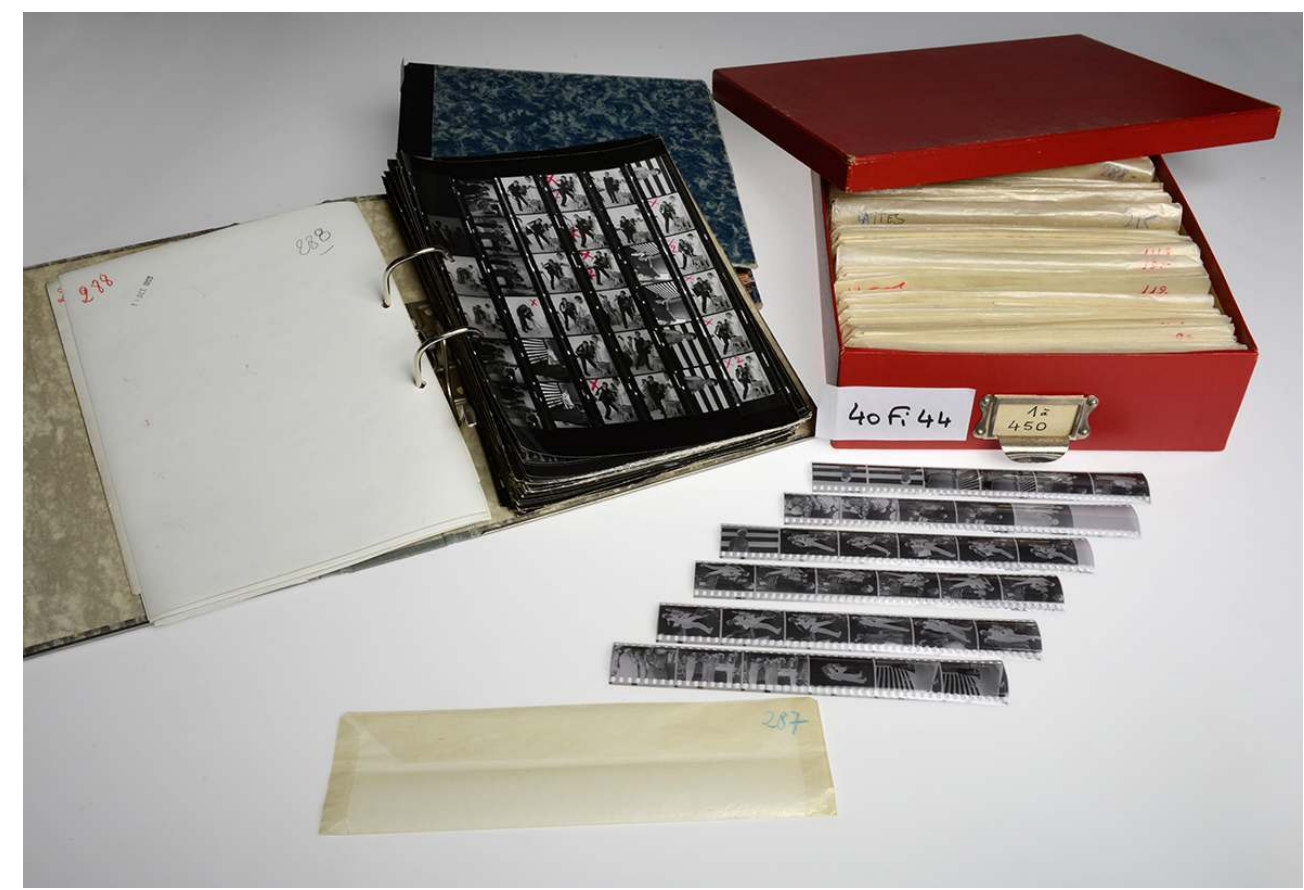

Négatifs et planches contact, fonds Jean Lattes (40 FI).

Phot. Jean-Bernard Barsamian. (C) Archives départementales des Yvelines, 2015.

12 Ces reportages, numérotés de 1 à 4934 pour les planches-contact et les bandes de négatifs et de $\mathrm{K} 1$ à $\mathrm{K} 333$ pour les diapositives couleurs ${ }^{14}$, ont fait l'objet d'un inventaire détaillé par Jean Lattes et sa femme.

Le troisième ensemble représente sa production au cours de l'aventure de l'agence Gamma, de 1966 à 1972. Celle-ci est clairement identifiée par la numérotation, précédée d'un G : 1858 planches-contact et autant de films de négatifs noir et blanc ( $24 \times 36)$ ainsi que 6400 diapositives couleurs. Contrairement aux deux autres ensembles, l'inventaire (sur simples fiches bristol) est très succinct mais les descriptions au dos des planchescontact ou sur les pochettes des clichés permettent aisément de retrouver leur contexte.

14 Un fichier matières qui compte plus de 2200 fiches cartonnées, désormais conditionnées en deux boîtes, est la clé de voûte du classement. Il correspond à la pratique traditionnelle utilisée en agence de presse avant l'introduction de systèmes documentaires informatisés pour organiser les fonds et offre un accès thématique unique à l'ensemble de la production photographique de Jean Lattes en tant qu'indépendant (fig. 5). 
Figure 5

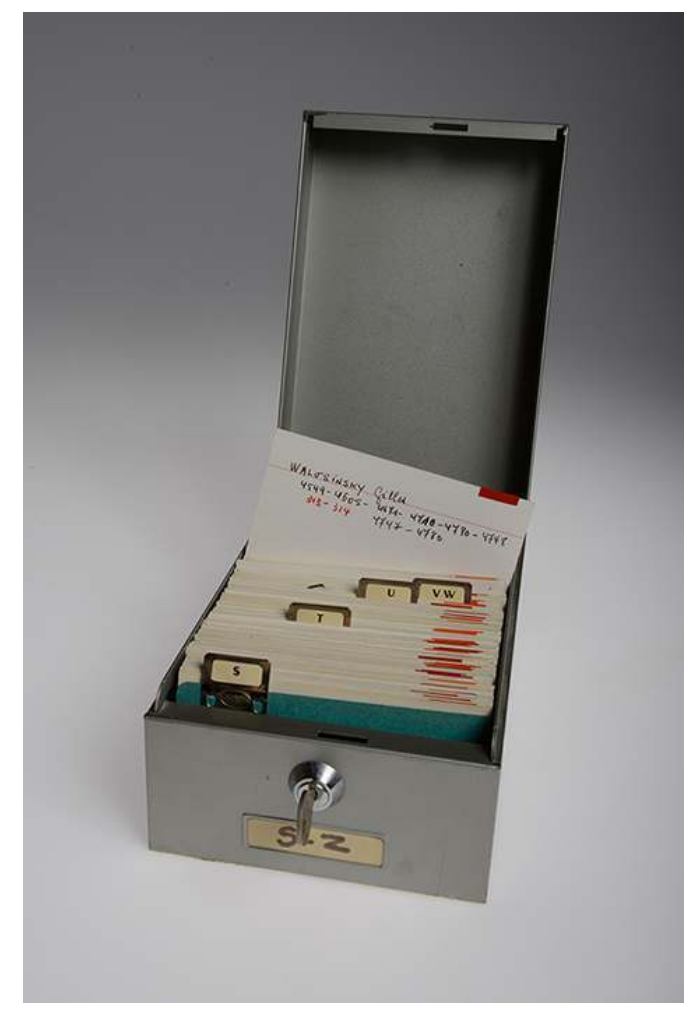

Fichier matière, fonds Jean Lattes (40 FI).

Phot. Jean-Bernard Barsamian. (C) Archives départementales des Yvelines, 2015.

Ce fichier, associé aux inventaires, permet de saisir la diversité des sujets, qui couvrent l'actualité politique, sociale et artistique des années 1950 à 1980 : événements et personnalités politiques (congrès de partis politiques de gauche, de Georges Marchais à François Mitterrand), conflits sociaux (Mai 1968, Lip, usines Renault), vedettes de la chanson française (Juliette Gréco, Georges Brassens, Johnny Halliday), du cinéma (Mireille Darc, Danielle Darrieux, Brigitte Bardot, Jane Fonda) ou littéraires (Françoise Sagan, Jean Cocteau), événements sportifs (24 Heures du Mans), culturels (ballets, concerts de jazz), reportages sur l'architecture et la mode. La production de Jean Lattes comporte également des photographies intimes, notamment de membres de la famille et d'amis, de vacances, des prises de vue d'expositions ou d'ouvrages auxquels il collabore (fig. 6, fig. 7). 
Figure 6

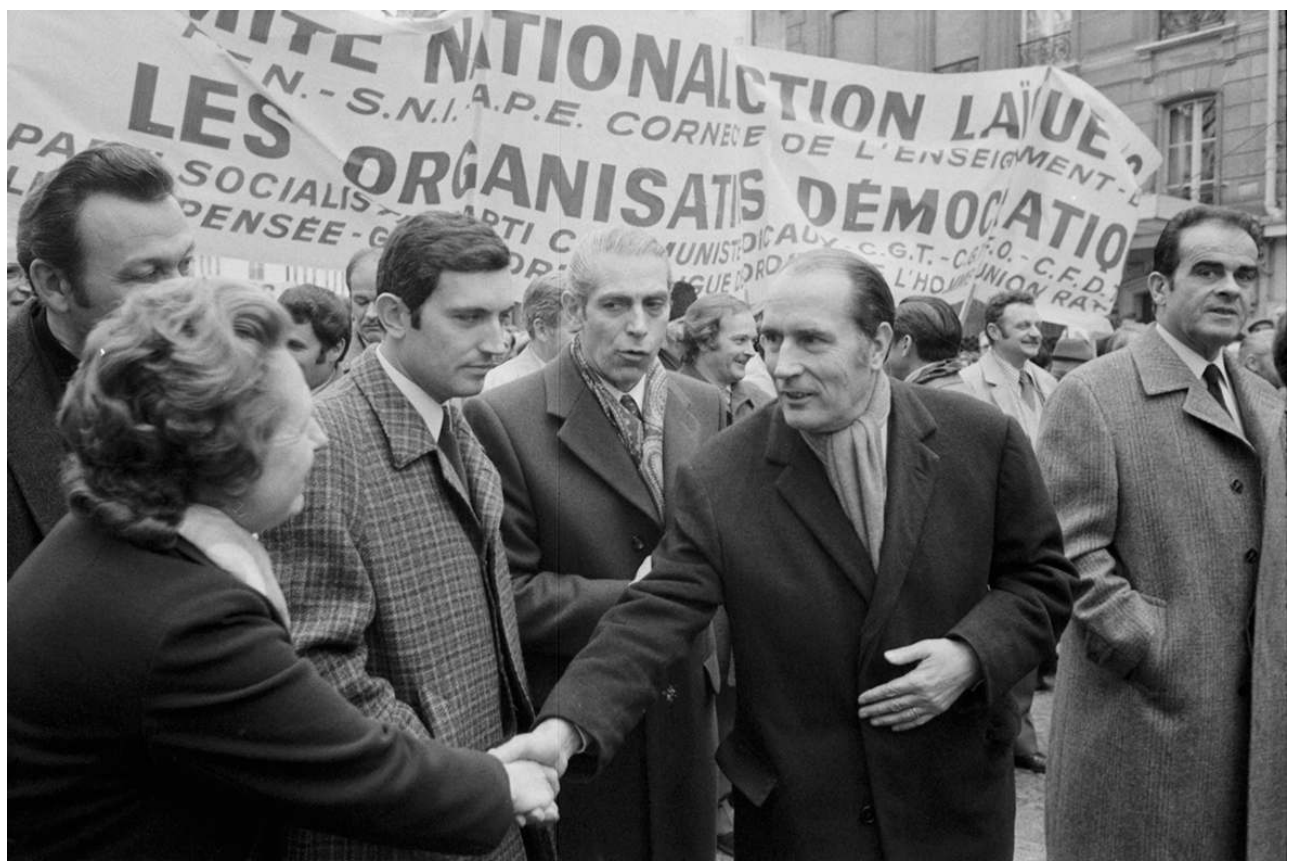

François Mitterrand, Comité National d'Action Laïque, 1972.

Phot. Jean Lattes. (c) Archives départementales des Yvelines, 2017.

\section{Figure 7}

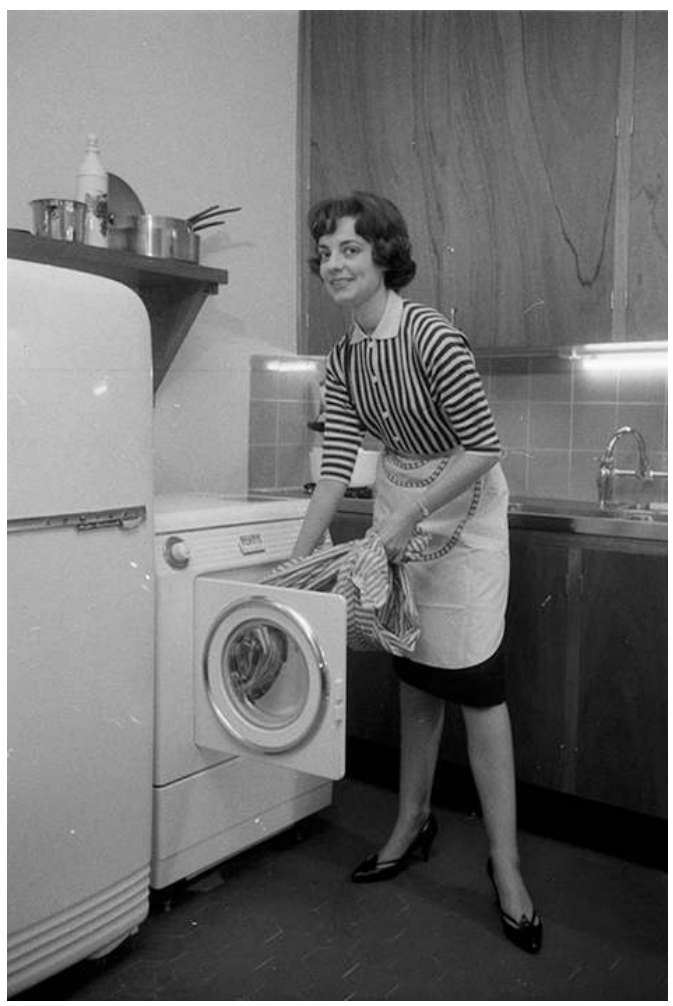

Bas Le Bourget, 1958.

Phot. Jean Lattes. ( ) Archives départementales des Yvelines, 2017. 


\section{Une vie de photographe}

16 1949. Le paysage de la presse française a alors été largement bouleversé par la figure emblématique de l'éditeur et directeur de plusieurs titres de presse Lucien Vogel (1886-1954). Ayant révélé plusieurs photographes dans les années 1930, dont Brassaï (1899-1984), Robert Capa (1913-1954) ou Man Ray (1890-1976), il a introduit avec les titres Femina puis $V u$ (1928-1940) la modernité dans les magazines illustrés ${ }^{15}$ par l'usage singulier qu'il accorde aux photographies, l'accent mis sur la qualité des images et le profond renouvellement de la mise en page. Jean Lattes cite volontiers l'influence de Lucien Vogel dans les autobiographies succinctes qu'il a rédigées pour expliquer son goût pour la photographie de presse ${ }^{16}$. célèbre agence est connue pour avoir défendu le droit d'auteur et son corollaire, la mention du nom du photographe à chaque publication ${ }^{17}$. La double localisation de Magnum à New York et à Paris donne un nouveau souffle à l'économie de la photographie de presse et favorise l'engagement du photographe dans son sujet et la maîtrise de la diffusion. Lattes inscrit sa pratique professionnelle dans ce sillage. Travaillant pour divers titres, il a veillé pendant sa carrière à rester propriétaire de ses négatifs. Les justificatifs conservés dans le fonds indiquent qu'il a ajouté a posteriori son monogramme pour signer ses images non créditées par le journal ou bien au contraire qu'il entoure son nom. Les deux agences qu'il a contribué à fonder, Gamma en 1967 et Viva en 1972, avaient comme caractéristique principale que le photographe restait propriétaire de ses négatifs. Cette condition est également stipulée pour un reportage de type sociologique et documentaire sur la ville de Nanterre commandité par la Bibliothèque publique d'information (BPI) ${ }^{18}$. Une telle exigence revêt un caractère déterminant dans la mesure où les négatifs ainsi appropriés et donc transmissibles constituent le cœur de la donation.

Jean Lattes a scrupuleusement conservé des traces comptables et administratives de son activité : contrats, attestations, justificatifs de publication, factures. Par exemple, en novembre 1950, alors qu'il était pigiste à France Dimanche, il obtient une modification de son contrat de travail pour que lui soit garanti un salaire fixe mensuel en contrepartie d'une exclusivité, "tout du moins en ce qui concerne les journaux ou hebdomadaire considérés comme des concurrents directs de France Dimanche $»^{19}$. Une étude précise de ces documents, couplée à celle des carnets de commande que le couple Lattes a tenus à jour, permettra à un chercheur ou un historien de la photographie une analyse sociale du métier de photoreporter pour au moins deux titres, France-soir et Elle (fig. 8).

In Situ, 36 | 2018 


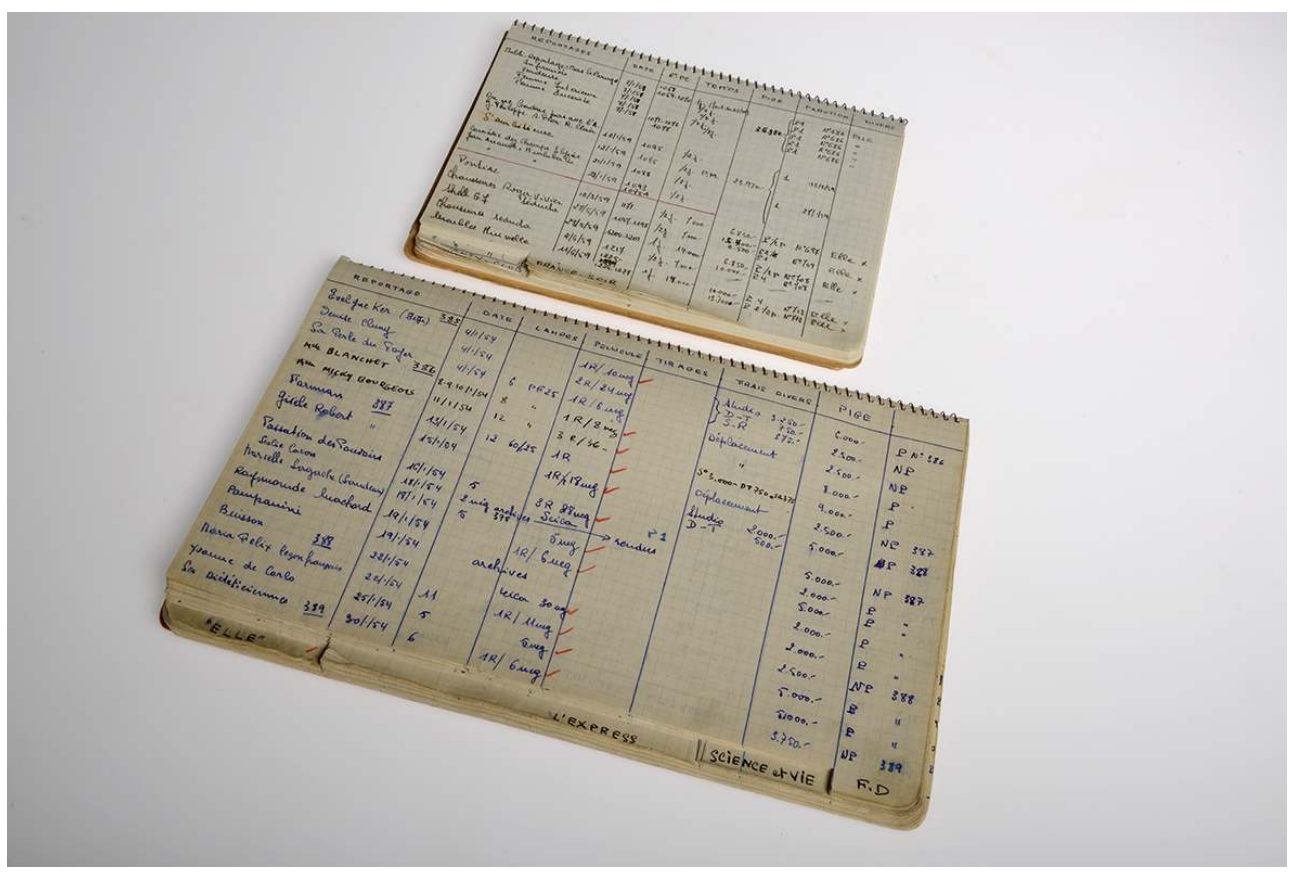

Carnets de commande, fonds Jean Lattes (40 FI).

Phot. Jean-Bernard Barsamian. (C) Archives départementales des Yvelines, 2015.

Le fonds comporte des justificatifs de publication (découpés) mais aussi des éléments disparates comme des tracts politiques, son ouvrage Sportphotographie, 1860-1960 ${ }^{20}$ et les diverses versions de son tapuscrit, des correspondances, des archives de ses expositions (plan de scénographies, cartons d'invitation), des coupures de journaux ${ }^{21}$, de multiples variations sur sa biographie ${ }^{22}$. En revanche, sa carte de presse, objet emblématique des journalistes, ne s'y trouve pas $^{23}$.

20 Un seul tirage dit « de presse » a été identifié à ce jour dans le fonds, artefact pourtant symbolique du fonctionnement d'une agence. Assimilé à un tirage de lecture destiné à diverses rédactions, il se caractérise par des éléments matériels facilement identifiables. Épreuve sur papier baryté jusque dans les années 1970, il est fortement contrasté ${ }^{24}$ et porte au verso diverses mentions, dont le tampon de l'agence ou celui du photographe. À ces mentions peuvent s'ajouter les indications de recadrage ou d'agrandissement mentionnées en pourcentage ou tracées sur l'épreuve. Le tirage identifié comme tel répond à ces critères, portant les tampons de l'agence Viva, le crédit du photographe, la mention Nouvelles littéraires et diverses indications pour l'agrandissement. La mise en vente, ces dernières années, de plusieurs clichés de Jean Lattes confirme qu'il était familier de cette pratique, comme on peut le voir avec un portrait de Françoise Sagan qui s'est vendu à 1100 euros chez Drouot en $2009^{25}$. Les autres tirages conservés sont des épreuves relatives à la commande passée en 1979 par le ministère de la Culture pour l'exposition « Dix photographes sur le patrimoine».

21 En revanche, les archives collectées ne permettent pas de comprendre le début de la chaîne commanditaire. Comment la commande est-elle formulée par les rédactions et adressée au photographe? Quels sont les conditions et les délais de réalisation imposés par le commanditaire? Comment s'effectue la transmission des informations aux rédactions et les échanges avec elles? Quelles sont les modalités matérielles du 
développement des films? Les échanges avec l'éditeur photo, qui sélectionne et met en page le travail du photographe, sont en effet centraux dans le fonctionnement d'une rédaction. De même, après la publication, si le circuit de distribution des images par Jean Lattes lui-même nous est inconnu, il est permis de penser qu'un système classificatoire si élaboré était précisément destiné à optimiser la recherche des images pour une diffusion ultérieure.

Les papiers administratifs sur sa participation à la fondation de l'agence $\mathrm{Viva}^{26}$ sont absents du fonds. Jean Lattes intègre Viva sous le pseudonyme de Jeremy Cartwright ${ }^{27}$, n'ayant pas encore quitté Gamma, mais aucun indice n'a été repéré dans les archives. De même, les photographies réalisées pour Familles en France ${ }^{28}$, le grand projet collectif de Viva, ne sont pas clairement identifiables dans le fonds. Enfin, même son rôle actif ${ }^{29}$ au sein de l'Association nationale des journalistes reporters photographes (ANJRP), révélateur de sa manière d'envisager le métier de photojournaliste, transparaît à peine.

\section{Le traitement du fonds : de l'analyse à la diffusion numérique}

Dès son entrée aux archives départementales des Yvelines, le traitement du fonds photographique de Jean Lattes a été jugé comme prioritaire avec comme finalité sa valorisation et sa diffusion numérique intégrale ${ }^{30}$ à un large public. Le fonds s'est construit autour de ses négatifs, supports fragiles considérés aujourd'hui en péril imminent. Dès lors, une course contre le temps s'est engagée pour leur conservation. Malgré l'urgence, le traitement s'avère long et débute sur quatre fronts presque simultanément: il faut d'une part mieux comprendre la production de cet ensemble représentatif de l'activité d'un homme dont nous venons d'exposer les différentes carrières. Ce travail chronophage est mené avec le concours de personnes reconnues pour leur expertise sur ce type de fonds et de proches de Jean Lattes encore en vie. D'autre part, la connaissance des reportages photographiques repose non seulement sur des inventaires et des fichiers tenus par le photographe mais aussi une myriade d'informations distillée dans le fonds, que ce soit sur les supports eux-mêmes ou leur conditionnement. Maîtriser intellectuellement l'ensemble nécessite de scruter chaque pièce avant d'entreprendre toute opération matérielle qui risquerait de faire perdre des données. Cette tâche accomplie, les premières mesures de conservation peuvent être prises, dès les opérations de reconditionnement des différents supports et leur cotation. Enfin, la numérisation, qui pourrait être vue comme une finalité dans la chaîne de traitement, intervient aussi en cours de classement comme nous le reverrons plus loin pour les Panodias, pochettes transparentes de conservation.

Pour la cotation, le fonds rejoint la série FI consacrée aux "documents figurés ", conformément au cadre de classement des archives départementales ${ }^{31}$. Afin de maintenir au mieux l'ordre originel de cet ensemble dont le classement n'appelle aucune réorganisation majeure, l'inventaire reprend les descriptions faites par son producteur. L'ensemble des métadonnées est ainsi ressaisi par un prestataire dans leur forme d'origine, là où elles se trouvent, que ce soit sur des fiches bristol pour les titres des reportages, sur le «fichier matières " pour toutes sortes d'éléments indexés, au dos des planches-contact pour les dates, voire sur les publications, les carnets de commande ou les pochettes en papier cristal quand on ne dispose d'aucune autre information. Une 
reprise de ces métadonnées se révèle indispensable par la suite, en particulier pour l'indexation. En effet, la valeur documentaire du corpus, particulièrement intéressante, n'est accessible que par la qualité de l'indexation. Celle-ci dépend de la connaissance du contexte historique de prise de vue. Cette question est d'autant plus cruciale que la recherche en sciences sociales s'appuie de plus en plus sur ces artefacts; «les images dites d'archives sont omniprésentes dans notre culture visuelle $\aleph^{32}$. L'indexation de Jean Lattes a ainsi été améliorée, normalisée et répartie en trois catégories : personne, lieu et thématique, événement.

Les réflexions archivistiques se heurtent systématiquement à cet ensemble fermé que constitue le film argentique. En général, l'unité intellectuelle (ici, la photographie ou le reportage photographique) est matériellement identifiable (un support, une pochette, un classeur, etc.). Regroupant de manière inhérente au moins 36 clichés successifs, le film argentique rassemble une suite chronologique de photos. Il peut contenir indifféremment un, plusieurs, ou une partie de reportages. Outre la difficulté d'analyse qui en découle, le classement de Lattes montre ses limites. Le photographe indexait au film; l'indexation pouvait concerner une ou plusieurs photographies sur les 36 . Celle-ci reste imprécise et seul l'auteur, à partir de sa planche-contact et en faisant appel à sa mémoire, pouvait identifier le sujet ou le lieu sur les clichés qu'il cherchait sur l'ensemble d'un film. Par ailleurs, dans son classement, Jean Lattes ne respectait pas la chronologie de ses prises de vue sur ses planches-contact et il en faisait probablement de même en rangeant pêle-mêle dans des pochettes cristal les sections de films développées. Ce désordre originel, joint aux défauts d'indexation, nuit à la fois à la compréhension des reportages et à l'identification des protagonistes. Là encore, une course contre le temps s'amorce car le photographe apporte un regard sur une génération de célébrités de plus en plus lointaines et donc de plus en plus difficiles à identifier.

Aucun fonds conservé par les archives départementales des Yvelines n'a nécessité autant d'anticipation dans l'articulation des différents traitements. $\mathrm{Ne}$ jamais perdre d'information rédigée par l'auteur et toujours pouvoir la rattacher aux documents comme à sa substitution numérique doit être l'obsession première avant toute intervention physique comme le reconditionnement des différents supports, leur cotation et leur numérisation. Celles-ci sont menées conjointement afin de limiter la manipulation des documents fragiles. L'état matériel préoccupant des négatifs impose un traitement spécifique. Conformément aux recommandations, ils sont conditionnés pièce à pièce dans des pochettes neutres et des boîtes adaptées. En revanche, le reconditionnement des diapositives soulève un autre problème : à défaut de planches-contact pour ce type de support, Lattes inscrivait l'analyse de ses diapositives sur des Panodias mais inadaptées à leur conservation. Une méthodologie est actuellement à l'étude pour permettre à la fois l'informatisation des données inscrites, la numérisation des pochettes avec les diapositives puis de chacune d'elles avant leur reconditionnement et leur cotation et enfin, le rattachement des analyses aux fichiers numériques (fig. 9). 


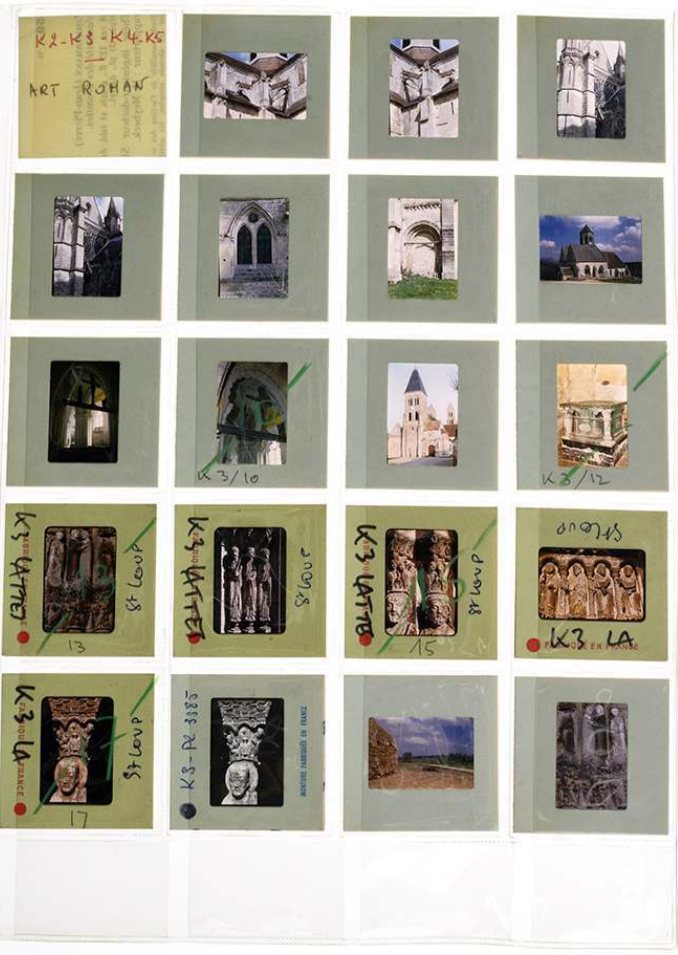

Diapositives, fonds Jean Lattes (40 FI).

Phot. Jean-Bernard Barsamian. (C) Archives départementales des Yvelines, 2015. négatifs et les planches-contact, recto et verso, puis les quelques tirages issus d'une sélection faite par l'auteur. L'objectif de la numérisation est à la fois de préserver le fonds mais aussi de connaître l'ensemble du processus de création de son producteur. Fonder notre campagne sur une sélection de clichés reste difficile dans la mesure où l'on ne dispose que de visuels dans un format miniature, voire uniquement des négatifs. Par ailleurs, dans la production d'un photojournaliste de cette envergure, les archivistes peuvent-ils s'arroger le droit de sélectionner l'image la plus adaptée pour illustrer un reportage, comme cela se pratique dans la photothèque d'un service ? Ce serait prendre le risque de trahir le regard de son auteur sur des événements ou des personnalités.

érisation, actuellement entreprise par un prestataire externe, fait l'objet d'un contrôle strict de qualité en interne. L'opération se fait sur des scanners équipés de passevues permettant d'aplanir les bandes et d'automatiser le cadrage qui peut au besoin être repris manuellement. Comme nous l'avons vu plus haut, ce passage au numérique de la production de Jean Lattes offre l'opportunité de sauter l'obstacle du support filmique présenté précédemment. Les clichés peuvent être réorganisés virtuellement par reportage et indexés individuellement. Ils suivent l'ordre chronologique des prises de vues indiqué sur les planches-contact. Ainsi, les trois supports qui sont offerts à la consultation en ligne dans une base de données reprenant les analyses de l'instrument de recherche portent des informations complémentaires : la planche-contact renseigne sur la chronologie et les post-traitements élaborés par le photographe, les images positives 
issues des négatifs ${ }^{33}$ sur le sujet dans son format brut et les quelques tirages sur les choix et les éventuels retraitements opérés par son auteur.

Alors qu'il est ami avec Henri Cartier-Bresson, Willy Ronis ${ }^{34}$ et Gisèle Freund ${ }^{35}$ et qu'il côtoie Robert Doisneau, Raymond Depardon et bien d'autres, force est de constater que Jean Lattes n'a pas endossé la figure mythique du photoreporter ${ }^{36}$ parcourant la planète. Il travaille à Paris, épicentre du photojournalisme à cette époque, mais malgré ce réseau et la participation à plusieurs expositions - dont le Salon national de la photographie à la Bibliothèque nationale $e^{37}$ - il demeure peu connu du grand public et est pratiquement ignoré des historiens de la photographie ${ }^{38}$. Parfaitement conscient de cela, Jean Lattes écrit dans une lettre adressée au journal Le Monde: «Peu m'importe d'être méconnu. Je suis de taille à le supporter : je suis un dissident ${ }^{39}$.» (fig. 10)

Figure 10

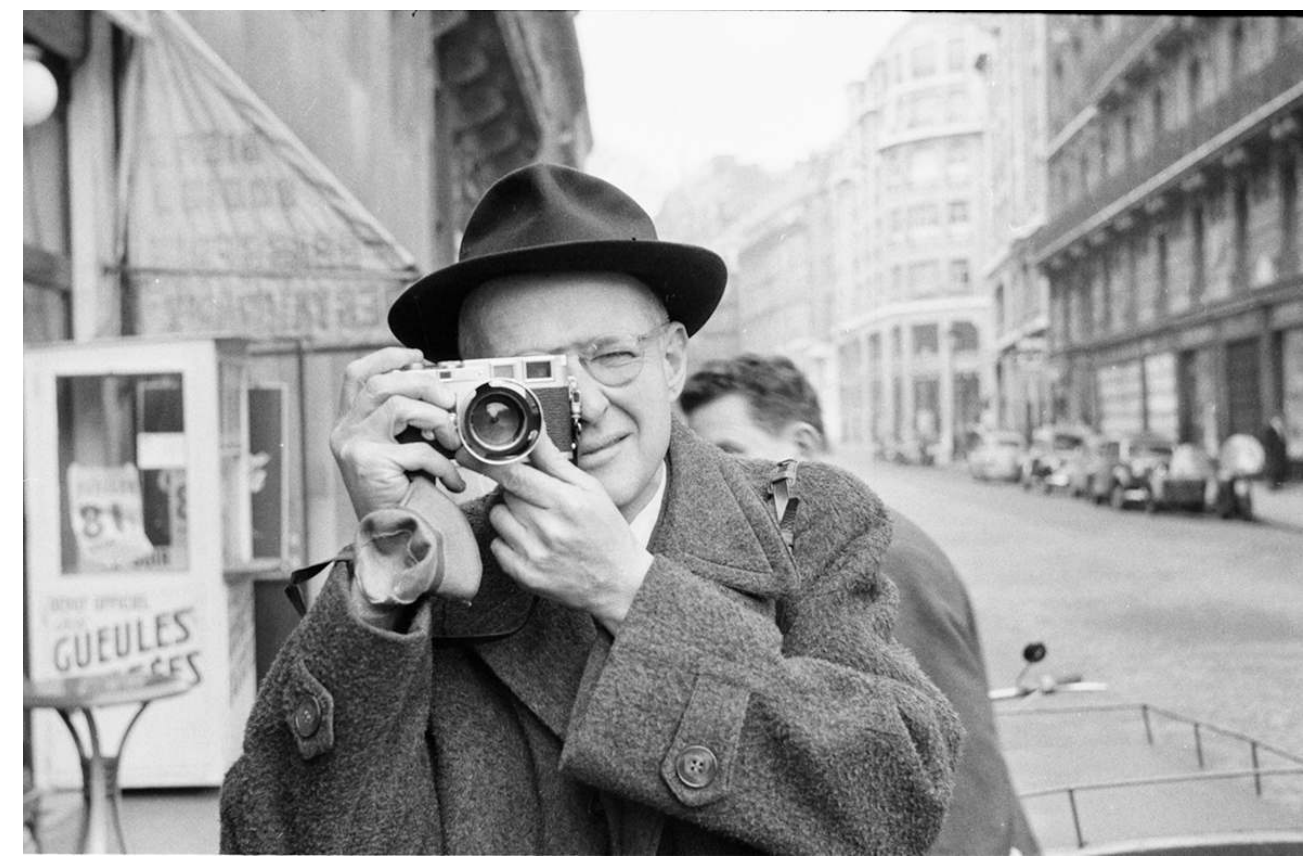

Henri Cartier Bresson, 1955.

Phot. Jean Lattes. (c) Archives départementales des Yvelines, 2017.

Si les éléments biographiques non transmis lors de la donation constituent des manques irrémédiables, l'archivage méthodique de son œuvre au fil de sa production a grandement facilité la compréhension de cette "archive continue", pour reprendre l'expression

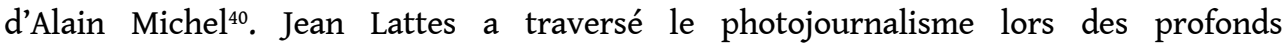
bouleversements du métier et du fonctionnement des agences. Ainsi, les archives versées sans tri préalable des clichés offrent-elles la possibilité d'une analyse par série des sujets comme de la pratique d'un photographe dont le statut a évolué. Bien que ces opérations d'analyse et de traitement du fonds vont se poursuivre sur plusieurs années encore, les archivistes imaginent déjà plusieurs formes de valorisation, au-delà de la mise en ligne, notamment sous la forme d'expositions. L'élaboration de celles-ci sera l'occasion de maintenir le travail déjà engagé en binôme entre archivistes et historiens de la photographie (fig. 11, 12, 13). 
Figure 11

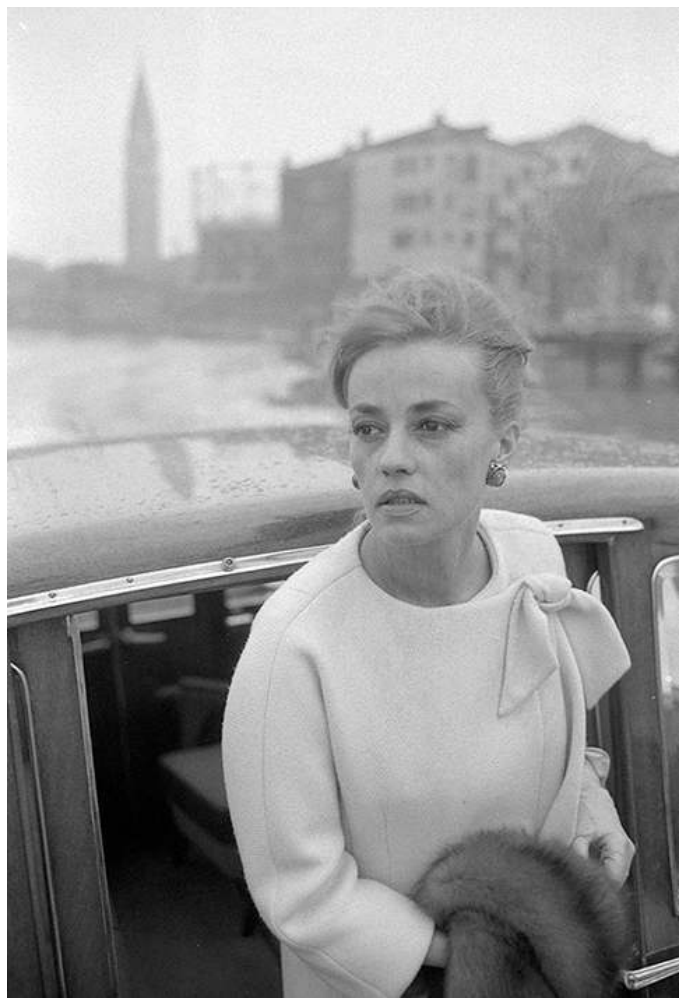

Jeanne Moreau,1961.

Phot. Jean Lattes. (C) Archives départementales des Yvelines, 2018.

Figure 12

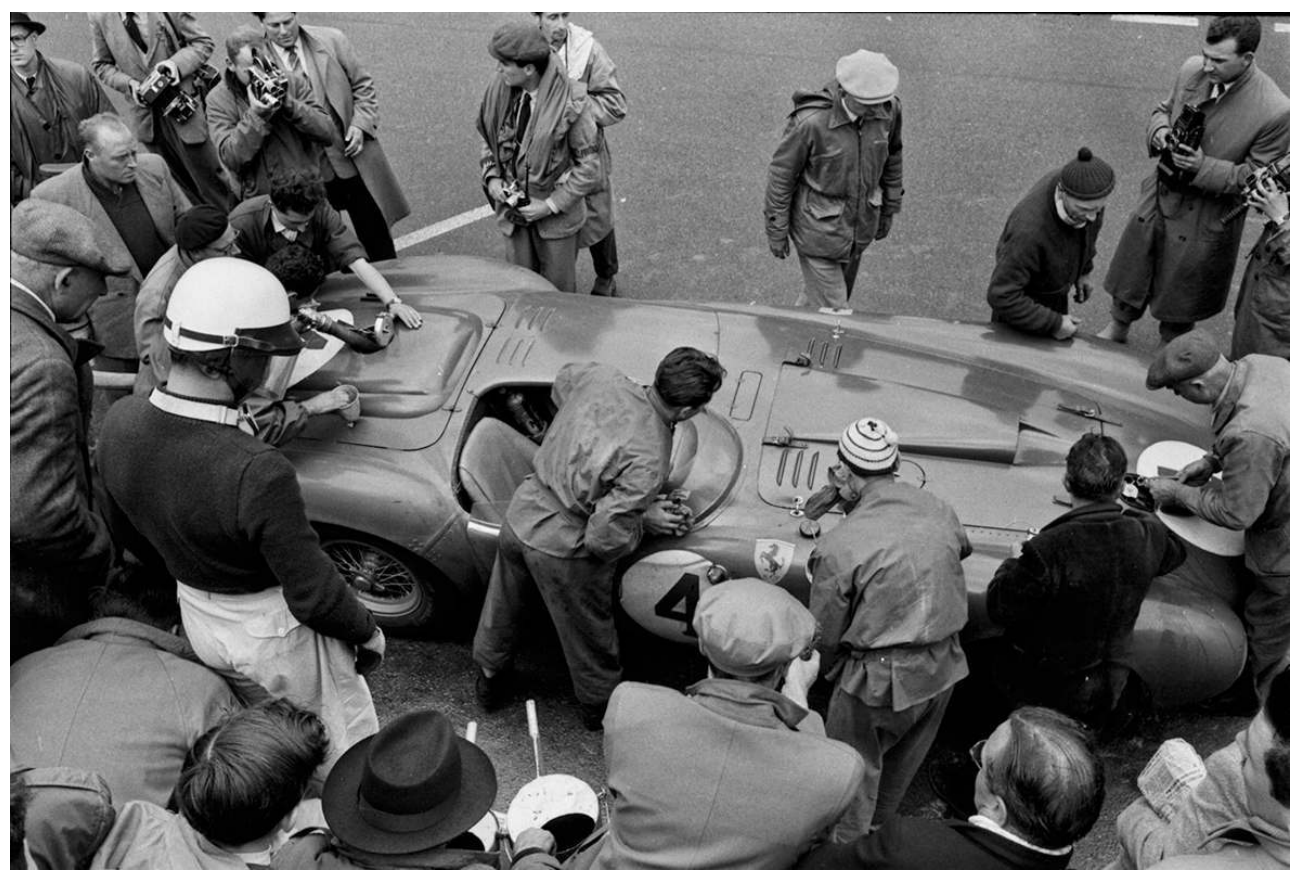

Les 24 heures du Mans, 1954

Phot. Jean Lattes. (c) Archives départementales des Yvelines, 2016. 
Figure 13

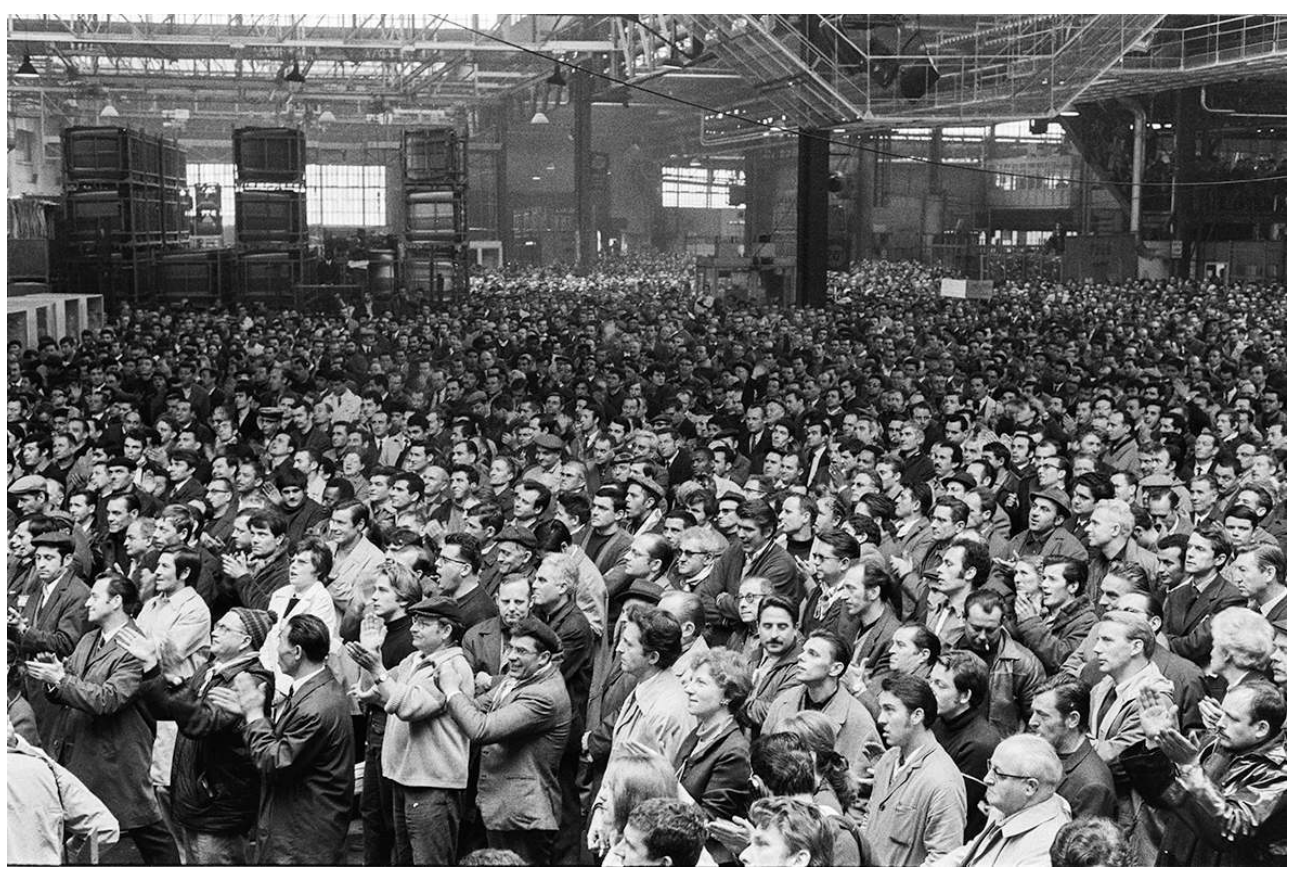

Grève chez Renault, 1968.

Phot. Jean Lattes. (c) Archives départementales des Yvelines, 2018.

\section{NOTES}

1. - Le nombre de clichés est approximatif et ne sera établi avec certitude qu'après le traitement et la numérisation du fonds.

2. - Depuis peu, une vacataire est chargée, aux archives départementales des Yvelines, d'établir un repérage systématique des fonds photographiques conservés.

3. - MARCILLOUX, Patrice. "Images archivées, images d'archives: fortunes terminologiques". Dans MAECK, Julie, STEINLE, Matthias (dir.). L'Image d'archives, une image en devenir. Rennes : PUR, 2016, p. 53-64.

4. - FERNANDEZ, Edmond. Aide (ou éléments d') à l'identification et à la préservation des photographies issues des procédés : photographiques, photomécaniques et électroniques. Paris : direction des Archives de France, 2014. Voir sur le site: http://www.culturecommunication.gouv.fr/content/ download/166308/1862812/version/2/file/

Annexe_2_DAF_BORA_PHOTO_AIDE_IDENTIFICATION_V15.pdf.

5. - La première édition de COLLARD, Claude, GIANNATTASIO, Isabelle, MELOT, Michel. Les Images dans les bibliothèques, est parue en 1995 aux Éditions du Cercle de la Librairie (édition revue en 2011, Images et bibliothèques).

6. - Le statut de la photographie de presse pose encore question : CHEVAL, François. «L'épreuve du musée ». Études photographiques, $\mathrm{n}^{0} 11$, mai 2002, p. 4-43 et MOREL, Gaëlle. Le Photoreportage 
d'auteur. L'institution culturelle de la photographie en France depuis les années 1970. Paris: CNRS Éditions, 2006.

7. - Colloque "Quel avenir pour les archives orphelines?», Archives nationales, Pierrefitte, 21 mars 2017.

8. - ARRAS, Marie-Noël, LATTES, Janine. Inoubliables. Femmes célèbres par Jean Lattes. Montpellier : Chèvre-feuille étoilée, 2011, p. 7.

9. - Les photographies de Jean Lattes représentaient, en $2015,70 \%$ du nombre de clichés conservés aux archives départementales des Yvelines.

10. - LAVÉDRINE, Bertrand. (re)Connaître et conserver les photographies anciennes. Paris : Éd. du CTHS, 2007, p. 266.

11. - Biographie rédigée par Jean Lattes à l'occasion de l'exposition « Dix photographes pour le patrimoine » présentée au Centre Georges Pompidou en 1980, (40FI).

12. - « Dix années d'indépendance (dans l'interdépendance, bien sûr!) pour Elle et le Nouveau Femina. La couleur entre dans ma vie, et je ne résume plus la vie des autres en une seule photographie ». Dans la biographie rédigée par Jean Lattes, op. cit.

13. - Réalisés avec un Leica ou d'autres appareils, ainsi que cela est parfois mentionné dans les carnets de commande du photographe.

14. - La lettre $\mathrm{K}$ fait probablement référence à la marque Kodak, les diapositives étant soit des Ektachromes soit des Kodachrome.

15. - DENOYELLE, Françoise. La lumière de Paris. Paris : L'Harmattan, 1997, t. 2, Les Usages de la photographie, p. 111.

16. - En hommage à Vogel, il a créé un magazine consacré à la photographie intitulé VU. Neuf numéros sont parus mais aucun n'a été versé aux archives départementales, bien qu'il écrivit à plusieurs reprises dans chaque numéro.

17. - La conquête du droit d'auteur commence en 1945 avec l'action de la National Press Photographers Association (NPPA) qui défend ardemment les crédits photographiques (voir LAVOIE, Vincent. Photojournalismes. Revoir les canons de l'image de presse. Paris : Hazan, 2010).

18. - Lettre de Jean-Pierre Seguin, conservateur en chef de la Bibliothèque publique d'information datée du 22 mai 1975, 40 FI.

19. - Lettre de Fernand Legouge, secrétaire général, du 21 novembre 1950, 40 FI.

20. - LATTES, Jean. Sportphotographie. Lucerne/Francfort-sur-le-Main : C. J. Bucher, 1977 (AD Yvelines, $40 \mathrm{FI})$.

21. - Ses coupures concernent autant son ouvrage que l'exposition «Dix photographes pour le patrimoine » qui s'est tenue au Centre Georges Pompidou en 1980, 40 FI.

22. - Les archives contiennent plusieurs versions de son autobiographie mais également des corrections sur des notices qui le concernent.

23. - L'attribution d'une carte de presse dépendait du statut du photographe. Certains pigistes y avaient droit, d'autres non. Nous ne savons pas si cette lacune du fonds est liée à une telle situation ou si ladite carte n'a tout simplement pas été versée.

24. - PACHE-ALLIMAN, Séverine. « De la dépêche au musée, quelques enjeux muséographiques ». Dans HAVER, Gianni (dir.). Photo de presse. Lausanne : Antipodes, Médias et Histoire, 2009, p. 92.

25. - « Françoise Sagan », estimation basse $300 €$, estimation haute $500 €$, prix au marteau $1100 €$, tirage argentique d'époque, $28,2 \mathrm{~cm}$ x 20,1 cm, circa 1970, lot n ${ }^{\circ} 93$ de la vente Lafon (S.V.V.), qui s'est tenue à Drouot-Paris. La vente Aponem, collection Peter Knapp, présentait des tirages montés sur carton et signés. Collection Peter Knapp - collection Mathilde Malaval et divers du 21 mars 2015 à Drouot-Richelieu. Il n'y a pas de trace de ces ventes dans les archives conservées.

26. - Jean Lattes n'est que rarement cité pour sa participation à la fondation de l'agence Gamma ; voir PUECH, Michel. Génération Gamma, Jean Lattes, le photographe oublié ( 2 épisode) ; dans l'enregistrement réalisé en 1975, Lattes explique le déroulement de la fondation de l'agence. Voir sur le site : https://www.youtube.com/watch?v=lFyjU2Ihchc [consulté le 30/08/2017]. 
27. - DELIGNY, Aurore. «Viva, une alternative à Magnum?». Études photographiques, 15, novembre 2004, [En ligne], mis en ligne le 27 octobre 2004. URL: http:// etudesphotographiques.revues.org/396 [consulté le 6/10/2017].

28. - Réalisée en 1973, cette enquête photographique collective de grande envergure a pris la forme d'une exposition itinérante. Conçu non sans difficulté, ce projet est désormais considéré comme le «manifeste » du groupe.

29. - Historique de l'ANJRPC, voir sur le site: http://www.upp-auteurs.fr/ profession_photojournaliste.php?section=qui-sommes-nous [consulté le 17/02/2018].

30. - Dans le respect du droit à l'image, ce qui n'est pas sans poser problème pour les personnes encore en vie présentes sur les clichés.

31. - Cette série FI existe depuis 1964.

32. - MAECK, Julie, STEINLE, Matthias. "On ne naît pas image d'archives, on le devient ». Dans L'Image d'archives, une image en devenir. Op. cit., p. 11.

33. - La visionneuse permettra de revenir à l'image en négatif.

34. - La correspondance entre Jean Lattes et Willy Ronis a été confiée par Janine Lattes au photographe Gilles Walusinski. Une seule lettre de Ronis, la première de la correspondance, a été versée dans le fonds. La particularité de cette correspondance est que Lattes tapait ses lettres à la machine à écrire et en conservait un double sur papier pelure.

35. - L'œil d'Henri Cartier-Bresson photographié par Jean Lattes illustre la couverture de l'ouvrage de référence, de FREUND, Gisèle. Photographie et société. Paris : éditions du Seuil, 1974.

36. - Figure décrite par CHÉROUX, Clément. «Mythologie du photographe de guerre ». Dans BLONDET-BISCH, FRANK, Robert, GERVEREAU, Laurent, et al. Voir, ne pas voir la guerre. Histoire des représentations photographiques de la guerre. Paris : Somogy/BDIC, 2001, p. 306-311.

37. - Il participe au moins à deux reprises, en 1959 et 1961, à ce salon inauguré en 1946.

38. - Le titre du billet du journaliste Michel Puech sur son blog «Jean Lattes, un photographe oublié » est révélateur. Voir sur le site : https://blogs.mediapart.fr/michel-puech/blog/241116/ generation-gamma-jean-lattes-le-photographe-oublie-2 [consulté le 11/09/2017].

39. - Le Monde ou Les infortunes de la photographie. Lettre tapuscrite adressée au journal Le Monde en réponse à la critique d'Hervé Guibert de l'exposition « Dix photographes et le patrimoine » (AD Yvelines, 40 FI).

40. - MICHEL, Alain P. «L'archive photographique, un document intégral ». Études photographiques , 16, mai 2005, [En ligne], mis en ligne le 17 septembre 2008. URL: http:// etudesphotographiques.revues.org/722 [consulté le 28/10/2017].

\section{RÉSUMÉS}

La donation de plus de 200000 clichés du photographe de presse Jean Lattes (1917-1996) en 2011 aux archives départementales des Yvelines, par sa femme Janine Lattes, a imposé aux archivistes d'engager une réflexion nouvelle sur leur pratique. En parallèle des préoccupations de conservation - bilan sanitaire, reconditionnement, campagnes de numérisation - se sont posées mécaniquement des questions liées au traitement intellectuel d'un ensemble photographique protéiforme, composé en outre d'archives relatives à son statut de pigiste, de cahiers d'inventaire et d'un fichier matière. La collecte précipitée, peu documentée à l'époque, a altéré la compréhension immédiate des pièces entre elles et des codes qui régissaient l'organisation 
initiale, conçue par les époux Lattes pour la gestion du fonds. Dès lors, la compréhension du contexte de production des clichés est apparue comme une nécessité pour élaborer des outils de recherche efficients, préserver les mentions du producteur et permettre une approche croisée des supports conservés - négatif, planche-contact et publication - en utilisant le reportage comme unité intellectuelle.

The donation in 2011, by his widow Janine Lattes, of more than 180,000 photos taken by the photojournalist Jean Lattes (1917 - 1996) to the archive service of the Yvelines department forced its archivists to reflect on their practice. In addition to immediate preservation concerns such as the physical state of the documents, reconditioning and digitising, other questions relating to the intellectual treatment of this complex photographic collection have also arisen. Besides photos, the collection also comprises archives relating to Jean Lattes's status as a freelance photographer, inventory books and a thematic catalogue. The transfer of the collection was a hasty affair, poorly documented at the time, and this affected the understanding of how the different documents relate to each other and of the codes that applied to the initial organisation, elaborated by the Lattes couple for the proper management of the collection. For this reason, understanding the context in which the photographs were produced seemed to be a preliminary necessity in order to conceive efficient research tools, to preserve the producer's own references and annotations and to allow a cross-cutting approach to the various media conserved, negatives, contact sheets and publications. For this the reportage was the most pertinent intellectual unit.

\section{INDEX}

Keywords : photo agency, departmental archives, classification, slide, donation, Jean Lattes, photography, negative, digitisation, contact sheet, press, Saint Quentin-en-Yvelines, press print Mots-clés : agence photographique, archives départementales, classement, diapositive, donation, Jean Lattes, photographie, négatif, numérisation, planche-contact, presse, SaintQuentin-en-Yvelines, tirage de presse

\section{AUTEURS}

\section{ANGELINA MESLEM}

Responsable de l'Unité de documentation, service des Musées de France, bureau de la Diffusion numérique des collections, direction générale des Patrimoines angelina.meslem@culture.gouv.fr

\section{WILFRID EON}

Chef du service Traitements des archives et diffusion numérique, pôle Archives, direction de la Culture, des Patrimoines et des Archives (D.C.P.A.) weon@yvelines.fr

\section{CLÉMENTINE VIALAR}

Adjointe au chef du service Traitements des archives et diffusion numérique, pôle Archives, direction de la Culture, des Patrimoines et des Archives (D.C.P.A.) cvialar@yvelines.fr 